\title{
An answer to a question by Wilf on packing distinct patterns in a permutation
}

\author{
Micah Coleman * \\ Submitted: Apr 21, 2004; Accepted: May 12, 2004; Published: May 24, 2004 \\ MR Subject Classifications: 05A05, 05A16
}

\begin{abstract}
We present a class of permutations for which the number of distinctly ordered subsequences of each permutation approaches an almost optimal value as the length of the permutation grows to infinity.
\end{abstract}

\section{Introduction}

Definition 1.1 Let $q=q_{1} q_{2} \ldots q_{k} \in S_{k}$ be a permutation, and let $k \leq n$. We say that the permutation $p=p_{1} p_{2} \cdots p_{n} \in S_{n}$ contains the pattern $q$ if there is a set of indices $1 \leq i_{1}<i_{2}<\cdots<i_{k} \leq n$ such that $p_{i_{1}}<p_{i_{2}}<\cdots<p_{i_{k}}$.

There has been significant interest in the topic of finding permutations containing many copies of the same pattern. In this paper, we will be concerned with the other extremity, permutations containing as many different patterns as possible.

At the Conference on Permutation Patterns, Otago, New Zealand, 2003, Herb Wilf asked how many distinct patterns could be contained in a permutation of length $n$. Based on empirical evidence, it seemed this number may approach the theoretical upper bound of $2^{n}$. In this paper we enumerate patterns contained in each of a certain class of permutations to at least establish a lower bound for this function.

Let $f(p)$ be the number of distinct patterns contained in a permutation $p$. Let $h(n)$ be the maximum $f(p)$, where the maximum is taken over all permutations of length $n$.

*Department of Mathematics, University of Florida, Gainesville FL 32611-8105. Supported by a grant from the University of Florida University Scholars Program, mentored by Miklós Bóna. 
On the one hand, $\sum_{k=0}^{n}\left(\begin{array}{l}n \\ k\end{array}\right)=2^{n}$ is an obvious upper bound for $h(n)$. On the other hand, there are only $k$ ! patterns of length $k$. So, for small $k$, we can replace $\left(\begin{array}{l}n \\ k\end{array}\right)$ with $k$ !. As $n$ grows, this second bound quickly becomes insignificant, as

$$
\left(\begin{array}{l}
n \\
k
\end{array}\right)<k !
$$

for all $k$ above a breakpoint which grows much slower than $n$.

Wilf demonstrated a class of permutations $W_{n}$ for which $f\left(W_{n}\right)$ asymptotically is greater than $\left(\frac{1+\sqrt{5}}{2}\right)^{n}$

Let $W_{n}$ denote the $n$-permutation $1 \quad n \quad 2 \quad n-1 \quad \cdots \quad\left\lceil\frac{n+1}{2}\right\rceil$. Let $W_{n}^{\prime}$ denote the $n$-permutation $n \quad 1 \quad n-1 \quad 3 \quad \cdots \quad\left\lfloor\frac{n+1}{2}\right\rfloor . W_{n}^{\prime}$ is called the complement of $W_{n}$. The $i^{t h}$ entry of $W_{n}$ is larger than the $j^{\text {th }}$ entry of $W_{n}$ if and only if the $j^{\text {th }}$ entry of $W_{n}^{\prime}$ is larger than the $i^{t h}$ entry of $W_{n}^{\prime}$. So, $W_{n}$ contains a pattern $q$ if and only if $W_{n}^{\prime}$ contains the complement $q^{\prime}$. Therefore,

$$
f\left(\begin{array}{llll}
p_{1} & p_{2} & \cdots & p_{n}
\end{array}\right)=f\left(n-p_{1}+1 \quad n-p_{2}+1 \quad \cdots \quad n-p_{n}+1\right)
$$

It should also be noted that the subsequence $W_{n}$ containing the $2^{\text {nd }}$ through $n^{\text {th }}$ entries is the complement of $W_{n-1}$. Then, the number of patterns contained in $W_{n}$ which do not include the first entry is $f\left(W_{n-1}^{\prime}\right)=f\left(W_{n-1}\right)$. The number of patterns contained in $W_{n}$ which are required to include the first entry but are distinct from those just enumerated is $f\left(W_{n-2}\right)$. If $f\left(W_{n}\right)=f\left(W_{n-1}\right)+f\left(W_{n-2}\right)$ then the sequence $W_{1}, W_{2}, \ldots$ is at least a Fibonacci sequence, where each point is the sum of the two previous points. In fact, this sequence has a rate of growth greater than the golden ratio $\frac{1+\sqrt{5}}{2}$, the (eventual) rate of growth of Fibonacci sequences.

In general, what can we say about $L=\lim \sup \sqrt[n]{h(n)}$ ? Wilf's result shows that $L \geq \frac{1+\sqrt{5}}{2}$. Our result will show that $L \geq 2$, so $L=2$. So, in this sense, our result is optimal. We establish this by presenting a class of permutations $\pi_{k}$ where $f\left(\pi_{k}\right)$ exceeds $2^{(k-1)^{2}}$.

We examined certain properties of all permutations up to length 10 and many beyond. A pleasantly surprising phenomenon was that $\frac{h(n+1)}{h(n)}$ appears to be a monotonically increasing function. The permutation

$$
512271510413811161439
$$

contains 16874 distinct patterns, more than $2^{n-1}$ for $n=15$. It seemed evident that Wilf's rate of growth could be improved upon.

\section{The Construction}

Definition 2.1 Let $p$ be a permutation. We call an entry $p_{i}$ a descent if $p_{i}>p_{i+1}$. 
Theorem 2.2 For $k \geq 2$, there exists a $k^{2}$-permutation containing more than $2^{(k-1)^{2}}$ distinct patterns

Proof: Let $\pi_{k}$ denote the permutation

$k 2 k \ldots k^{2}(k-1)(2 k-1) \ldots\left(k^{2}-1\right) \quad \ldots \ldots \quad 1(k+1)(2 k+1) \ldots\left(k^{2}-k+1\right) \in S_{k^{2}}$

For example,

$$
\begin{aligned}
& \pi_{3}=369 \quad 258 \quad 147 \\
& \pi_{4}=481216 \quad 371115 \quad 261014 \quad 15913
\end{aligned}
$$

It should be noted that the only descents in such a permutation are at the last entry of each segment, descending to the first entry of the subsequent segment. As these points play a significant role in our proof, we shall denote the first entry of each segment the base of that segment.

Also, each segment is structured so that the $i^{\text {th }}$ entry of that segment is less than the $i^{t h}$ entry of each preceding segment.

As counting all patterns of such a permutation leads to overwhelming complexity, we will restrict our attention to counting only certain patterns. Let $k \geq 2$. For the subsequences under consideration, we require that the first $k$ entries of $\pi_{k}$ be included, i.e., every entry in the first segment. Also, we include the base of each of the other segments. Considering $\pi_{4}$ as an example, we require the following underlined entries to be included.

$$
\underline{4} \underline{8} \underline{12} \underline{16} \quad \underline{3} 71115 \underline{2} 61014 \quad \underline{1} 5913
$$

Requiring these $2 k-1$ entries leaves $k^{2}-(2 k-1)=(k-1)^{2}$ entries remaining to choose from. There are $2^{(k-1)^{2}}$ subsets of these remaining entries. We claim that the subsequences each of which is the union of the required entries and some subset of these remaining entries are distinctly ordered, i.e., correspond to distinct patterns.

Suppose $q=q_{1} q_{2} \ldots q_{m}$ and $r=r_{1} r_{2} \ldots r_{m}$ are identically ordered subsequences of this type, both of length $m$. Then, the descents in $q$ and $r$ must be at the same positions. We required the base of each segment of $\pi_{k}$ to be included in $q$ and $r$, so any descent will immediately precede a base. Therefore, each base occupies the same position in $q$ as in $r$. This, in turn, implies that the $i^{t h}$ entry of $q$ lives in the same segment of $\pi_{k}$ as does the $i^{t h}$ entry of $r$, since they are situated between the same bases.

Furthermore, included in both $q$ and $r$ are all entries of the first segment of $\pi_{k}$. Since $q$ and $r$ are identically ordered, by definition, $q_{i}<q_{j} \Longleftrightarrow r_{i}<r_{j}$. If there was some entry in the first segment of $\pi_{k}$ that was less than some $q_{i}$ and greater than $r_{i}$, then $q$ and $r$ would not be distinctly ordered as was assumed. As noted earlier, the $i^{\text {th }}$ entry of each 
segment of $\pi_{k}$ is less than the $i^{t h}$ entry of $\pi_{k}$ itself. So, for all $i, q_{i}$ and $r_{i}$ occupy the same position within the same segment and are, in fact, equal. Therefore, $q$ coincides with $r$.

We've shown that two identically ordered subsequences must actually be the same, and our claim follows that the $2^{(k-1)^{2}}$ such subsequences constitute $2^{(k-1)^{2}}$ distinct patterns. Therefore, $f\left(\pi_{k}\right)=2^{(k-1)^{2}}$ for all $k \geq 2$.

\section{Long Distance Relationships}

There is still much in this area to be explored. While the above class of permutations lends itself to proof, like Wilf's, it is a tradeoff between manageability and performance. We have only counted a restricted number of patterns in certain less than optimal permutations. The Holy Grail here would be tighter bounds for $h(n)$.

A preferable result would be an inductive proof on $n$ that for any permutation $\pi$ of length $n$, one could find a permutation of length $n+1$ that contains $\pi$ as well as at least $2 f(\pi)$ patterns. This, with the upper bound, would be a clean proof that $h(n)$ grows asymptotically as $2^{n}$ and allow for more understanding of how $h(n)$ grows.

The aim for optimizing the permutation is typically to maximize the sum of the geographical and numerical distances between any two entries. For example, if $q_{i+1}=q_{i}+1$ in a given permutation, then any subsequence $q_{j_{1}} \cdots q_{i} \cdots q_{j_{k}}$ would correspond to the same pattern as would $q_{j_{1}} \cdots q_{i+1} \cdots q_{j_{k}}$. Maximizing the distances between entries minimizes this sort of waste. That was how the above class of permutations was discovered, although this property was not explicitly used in the proof.

\section{References}

[1] M. H. Albert, M. D. Atkinson, C. C. Handley, D. A. Holton, W. Stromquist, On packing densities of permutations. Electron. J. Combin., 9(1) (2002), R5.

[2] M. Bóna, B. E. Sagan, V. Vatter, Frequency sequences with no internal zeros. Adv. Appl. Math, 28 (2002), 395-420.

[3] D.Warren, Optimal Packing Behavior of some 2-block Patterns. Preprint, arXiv, math.CO/0404113. 